\title{
Bone Morphogenetic Protein-Transduced Human Fibroblasts Convert to Osteoblasts and Form Bone in Vivo
}

\author{
R. BRUCE RUTHERFORD, D.D.S., Ph.D., ${ }^{1}$ MARIA MOALLI, D.V.M.,, \\ RENNY T. FRANCESCHI, Ph.D., ${ }^{1}$ DIAN WANG, M.S., ${ }^{1}$ KENI GU, M.D., 1 \\ and PAUL H. KREBSBACH, D.D.S., Ph.D. ${ }^{1}$
}

\begin{abstract}
Experimental cell or ex vivo gene therapy for localized bone formation typically uses osteoprogenitor cells propagated from periosteum or bone marrow. Both require bone or marrow biopsies to obtain cells. We have demonstrated that implantation of gingival or dermal fibroblasts transduced with BMP ex vivo, using a recombinant adenovirus (AdCMVBMP) attached to porous biodegradable scaffolds, form bone in vivo. Here we show that BMP-7transduced fibroblasts suspended in injectable thermoset hydrogels form complete ossicles on subcutaneous injection and repair segmental defects in rat femurs. Bone formation was preceded by an intermediate cartilage stage. To determine the fate of the implanted transduced cells, thermoset hydrogel suspensions of ex vivo BMP-7-transduced or nontransduced fibroblasts were placed in diffusion chambers and implanted to allow development in vivo without direct contact with host cells. Only the BMP-transduced fibroblasts formed bone within the diffusion chambers in vivo, revealing that BMP transduction induces osteoblastic conversion of these cells.
\end{abstract}

\section{INTRODUCTION}

\begin{abstract}
Cell therapy, one current approach to tissue engineering, may be utilized for the restoration of congenital or acquired loss of tissue structure and function. In a combination of cell and gene therapy, known as ex vivo gene therapy, ${ }^{1}$ the cells to be implanted are removed from the patient and genetically altered before implantation in vivo. The genetically altered cells have typically been fibroblasts or myoblasts that are easily biopsied and propagated in vitro. ${ }^{2}$ Genetic alteration may be accomplished with recombinant plasmid or viral vectors.

Osteoprogenitor cells such as those derived from bone marrow cultures or periosteum form bone on implantation in vivo without ${ }^{(3,4)}$ or with ex vivo genetic modification. ${ }^{5-13}$ However, this approach requires that bone or bone marrow be biopsied; a procedure often associated with substantial donor site morbidity. Obtaining cells for autologous grafts from easily biopsied, readily regenerating tissues such as skin or gingiva would likely be associated with less morbidity. Therefore we have utilized ex vivo gene therapy to test the idea that autologous cells for tissue regeneration need not be obtained from the tissue to be regenerated. ${ }^{14}$
\end{abstract}

\footnotetext{
${ }^{1}$ School of Dentistry, Center for Biorestoration of Oral Health, and ${ }^{2}$ School of Medicine, Department of Orthopedic Surgery, University of Michigan, Ann Arbor, Michigan.
} 
This approach was suggested by the well-documented capacity of recombinant human bone morphogenetic proteins (BMP) to induce endochondral-like bone formation on implantation into dermis, muscle, ${ }^{15,16}$ or gingiva (R.B. Rutherford, unpublished observations) and the evidence that local cells initiate healing responses (reviewed by Prokop ${ }^{17}$ ). These observations suggest that several tissue types contain populations of cells responsive to the inductive effects of BMP. We have demonstrated that cultured nonimmortal strains of gingival or dermal fibroblasts, transduced ex vivo by the mouse BMP-7 full-length cDNA, secrete biologically active BMP-7 in vitro, and when absorbed to porous, preformed sponges and implanted in vivo, induce ectopic and orthotopic bone formation. Both the implanted donor and host cells participate in the bone formation, resulting in chimeric ossicles comprising both donor and host-derived extracellular matrix. ${ }^{14}$ These data suggested that the BMP-transduced fibroblasts in contact with host tissue converted to osteoblasts in vivo.

The use of BMP-transduced fibroblasts that contribute to bony matrix production as well as induce host tissues to produce bone could be advantageous in clinical conditions characterized by relatively large acellular lesions such as those produced by surgical resection of tumors and radiation therapy. Our hypothesis is that BMP-responsive fibroblasts may be propagated from skin and gingiva ex vivo and stimulated to form predetermined amounts of bone on implantation in vivo. Here we report that BMP-transduced fibroblasts suspended in thermoset hydrogels from cartilage that converts to marrow-containing ectopic ossicles in vivo. The size of these ectopic ossicles varies with the number of cells injected. Such preparations absorbed to preformed porous sponges also induce repair of femoral segmental defects. In addition, BMP-transduced fibroblasts suspended in a hydrogel form bone in vivo in the absence of direct contact with host tissue. These data reveal that BMP-7 transduction induces an osteoblastic conversion of nonosteoblastic fibroblasts that is not dependent on direct interaction of the transduced cells with host tissue.

\section{MATERIALS AND METHODS}

\section{Materials}

All reagents were purchased from Sigma (St. Louis, MO) or GIBCO-BRL (Grand Island, NY) unless otherwise noted. Defined fetal bovine sera were obtained from HyClone (Provo, UT). Gelfoam was obtained from Upjohn (Kalamazoo, MI) and tissue culture ware was obtained from Corning (Corning, NY). NIH-bg-nu-xid br (NIH III) and C57BL/6 mice were purchased from Charles River (Wilmington, MA).

\section{BMP transduction and preparation of cell-hydrogel suspensions}

Human gingival (HGF), C57BL/6 mouse dermal (MDF), or Fischer rat (RDF) fibroblastic cell strains were derived from explants of tissue biopsies and cultured as described. ${ }^{18}$ Cells from passage $3-12$ were removed from liquid nitrogen storage and grown to near confluence in Dulbecco's modified Eagle's medium (DMEM) supplemented with $10 \%$ fetal calf serum, penicillin, and streptomycin. HGF infection with AdRSVlacZ or with AdCMVBMP-2, -4 , or $-7^{19}$ was at a multiplicity of infection (MOI) of 200 PFU/cell whereas MDF and $\mathrm{RDF}$ infection was at $1000 \mathrm{PFU} /$ cell. Cells, confluent in $75-\mathrm{cm}^{2}$ flasks, were exposed to the appropriate dilution of virus in $8 \mathrm{ml}$ of medium for $24 \mathrm{~h}$ at $37^{\circ} \mathrm{C}$ in a humidified atmosphere of $5 \% \mathrm{CO}_{2}$ before trypsinization, counting, and resuspension in the appropriate hydrogel at the desired concentration. Matrigel (growth factor reduced; Collaborative Biomedical Products, Bedford, MA) and acid-soluble rat tail collagen solution (final concentration, $2.5 \mathrm{mg} / \mathrm{mL}$; Collaborative Biomedical Products) were prepared according to the manufacturer directions. Pluronic F127 (BASF, Mt. Olive, NJ) was mixed in sterile distilled water to a final concentration of $30 \%$. All these materials are thermoset hydrogels at the concentrations used: liquids at $4^{\circ} \mathrm{C}$ that gel as the temperature approaches $37^{\circ} \mathrm{C}$. The mass of ossicles was determined by wet weight and the data were analyzed by analysis of variance (ANOVA) and post-hoc analysis for significance with the Scheffe F test. The student $t$ test for paired samples was also used where appropriate.

\section{Implantation of transduced cells and tissue analysis}

Transduced cells were transplanted via subcutaneous injection of $200 \mu \mathrm{L}$ of the indicated number of cells in the backs of anesthetized NIH III mice for HGFs, or of C57BL/6 mice for MDFs. For the injections, a 26- 
gauge hypodermic needle was used. The C57BL/6 cells and host animals are syngeneic, hence this system models autologous transplantation into an immunocompetent host. For diffusion chamber experiments, $30 \mu \mathrm{L}$ containing a $10^{5}$ BMP-7-transduced or nontransduced HGF-collagen suspension was injected into 4-mm-diameter chambers prepared in sterilized $14 \times 2 \mathrm{~mm}$ custom plastic disks and sealed with nitrocellulose membranes $(0.45 \mu \mathrm{m}$; Millipore, Bedford, MA). The sealed diffusion chambers were implanted subcutaneously.

For orthotopic assays of bone formation, Fisher rats were anesthetized with isoflurane and the hindlimbs were aseptically prepared with Betadine and alcohol. The femurs were surgically exposed and a custom drill template was used to drill and insert four fixation pins into the femur. An external fixator with a central hinge, locked in a neutral position, was secured on the pins. A transverse simple 2- to 3-mm osteotomy was created midway between the central two fixator pins with a micro-oscillating saw. The BMP-7transduced RDF-collagen construct was inserted into the osteotomy site and the soft tissues were closed. BMP-7-transduced RDF-collagen construct comprised $10^{4}$ cells suspended in type I collagen hydrogel (2.5 $\mathrm{mg} / \mathrm{mL}$, final concentration) and absorbed to moistened and flattened precut Gelfoam sponges. Control constructs contained $10^{6}$ lacZ-transduced RDF.

After the indicated developmental interval in vivo, the tissues were harvested and fixed in fresh $4 \%$ paraformaldehyde, demineralized in $10 \%$ formic acid, and processed for histological or immunocytochemical analysis. Immunocytochemical analysis for BMP-7 expression was performed according to the Vectastain ABC kit protocol (Vector Laboratories, Burlingame, CA). BMP-7 ligand and antibody were purchased from Santa Cruz Biotechnology (Santa Cruz, CA). For calcium analysis, the ossicles were blotted dry after harvest, weighed, and incubated at $400^{\circ} \mathrm{C}$ for at least $8 \mathrm{~h}$. The ash was weighed and analyzed for calcium content according to the manufacturers specifications (kit 587A; Sigma). The photomicrographs taken with a digital camera were altered for brightness and contrast only in Adobe Photoshop (version 5.0). All procedures using animals were performed under protocols approved by the University of Michigan Committee on the Use and Care of Animals.

\section{RESULTS}

\section{Ectopic ossicle formation by injected BMP-transduced fibroblast-hydrogel suspensions}

Human gingival fibroblasts (HGFs), transduced ex vivo with a recombinant adenovirus containing the full-length mouse BMP-7 cDNA, ${ }^{19}$ formed marrow-containing ossicles in vivo when suspended in acidsoluble type I collagen and injected subcutaneously (Fig. 1). All ossicles produced by injecting BMP-2, 4, or -7-transduced HGFs revealed a similar histological structure (Fig. 1A,B). However, nontransduced HGFs, or HGFs transduced with bacterial lacZ, failed to form ossicles (Fig. 1C) and consistently produced identifiable tissue masses only after large numbers of cells were implanted.

The mass of the ossicles varied with the number of BMP-7-transduced HGFs injected when suspended in any of three distinct types of thermoset hydrogel (Table 1). Matrigel is a mixture of type IV collagen, laminin, and trace amounts of growth factors. The type I collagen is an acid-soluble fraction extracted from rat tails and Pluronic F127 is a surfactant with the common chemical name polyethylene-polyoxypropylene block copolymer previously demonstrated to be effective as a cell delivery vehicle. ${ }^{20}$ Increasing the number of cells $\left(10^{3}\right.$ to $\left.2 \times 10^{6}\right)$ injected in a constant volume of hydrogel $(200 \mu \mathrm{L})$ yielded larger ossicles in both immunodeficient and immunocompetent hosts (Table 1). As few as $10^{3}$ and $10^{4}$ BMP-transduced cells suspended in Matrigel or collagen, respectively, formed complete ectopic ossicles (Fig. 1 and Table 1) on subcutaneous injection. The mass of the ossicles produced by equal numbers of cells was greatest in Matrigel (Table 1). All ossicles, regardless of hydrogel carrier or cell number injected, were composed of cortical and medullary bone and contained marrow (Fig. 1). The calcium content of the ossicles produced by BMP-7-transduced HGFs suspended in collagen increased with increasing numbers of implanted transduced cells and was significantly higher than tissue formed in lacZ-transduced cell implants (Fig. 2).

\section{BMP-transduced fibroblasts induce cartilage and bone}

Implantation of exogenous BMP induces an endochondral-like bone formation process. ${ }^{21}$ To determine whether ossicles produced by BMP-7-transduced fibroblasts follow a similar developmental pathway, in- 


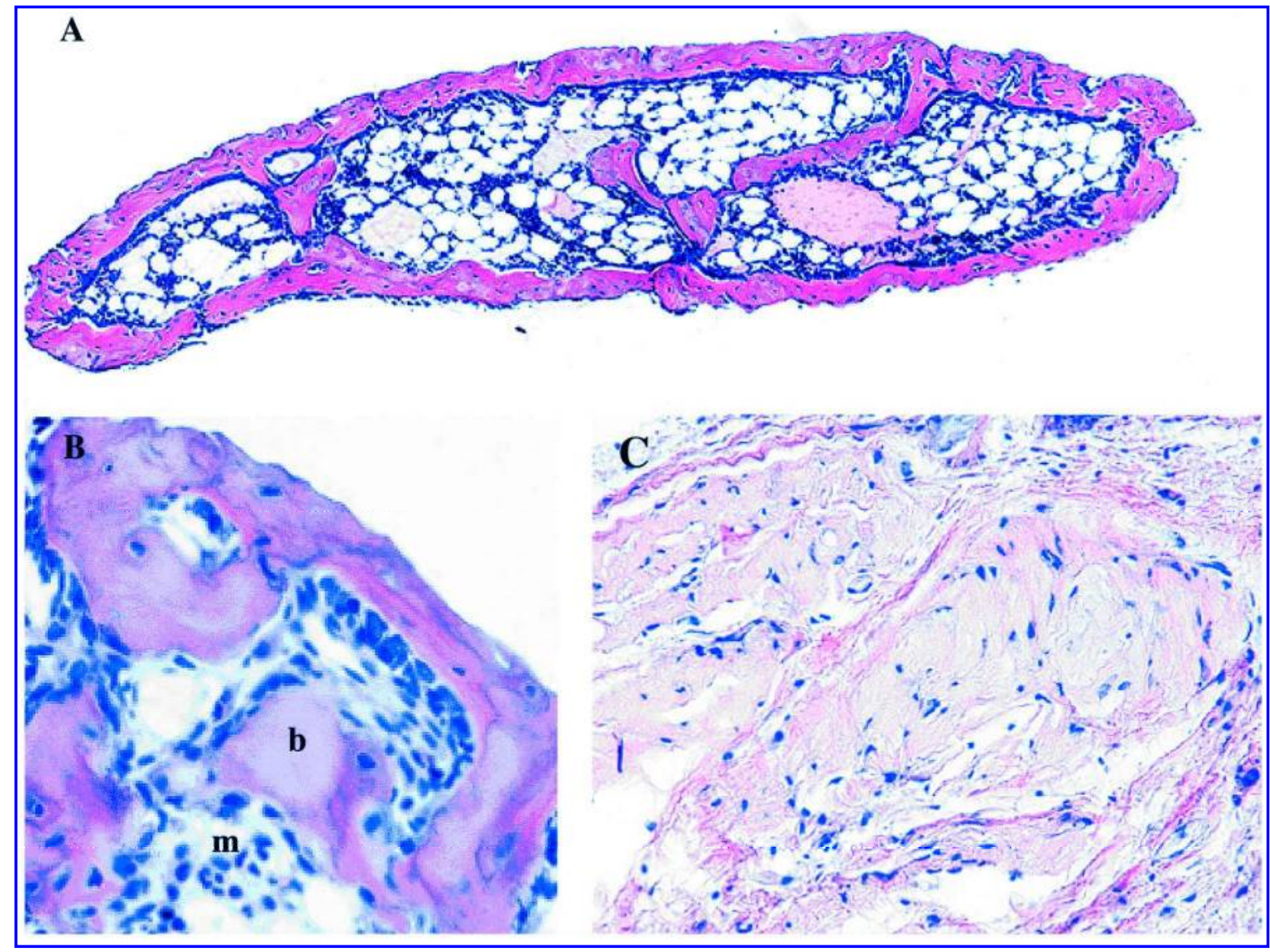

FIG. 1. BMP-transduced fibroblasts suspended in a collagen thermoset hydrogel form ectopic marrow-containing ossicles in vivo. BMP-7-transduced $(\mathbf{A}, \mathbf{B})$ or nontransduced $(\mathbf{C}) \mathrm{HGFs}$ were suspended in soluble thermoset collagen hydrogel $(2.5 \mathrm{mg} / \mathrm{ml}$, final concentration), injected subcutaneously into NIH III mice, harvested after 3 weeks, and analyzed histologically. (A) Histomicrograph of a typical ossicle formed with BMP-7-transduced HGFs suspended at $10^{4}$ cells $/ 200 \mu \mathrm{L}$ of collagen; original magnification, $\times 10$. (B) Higher power magnification of sample in $(\mathbf{A})$, demonstrating bony trabeculae (b) and marrow (m); original magnification, $\times 50 ;(\mathbf{C})$ histomicrograph of tissue produced by injection of lacZ-transduced, collagen hydrogel-suspendedHGFs original magnification, $\times 50$. All sections were stained with H\&E.

duced tissues were harvested at various intervals and histological specimens were prepared. Histochemical analyses of tissues produced by subcutaneous injection of $5 \times 10^{5} \mathrm{C} 57 \mathrm{BL} / 6$ fibroblasts into syngeneic hosts reveal that cartilage precedes bone $(n=4 / 4$; Fig. 3$)$. After 3 days, a condensation of cells is present (Fig. 3A) that matures to cartilage by 5-7 days (Fig. 3B) and to bone by 14 days (Fig. 3C). The cartilage phenotype is confirmed by the presence of cartilage extracellular matrix proteoglycans as detected by Safranin O staining after 6 days in vivo (Fig. 3B). By 14 days developing marrow is evident (Fig. 3C, arrow).

\section{Skeletal defect repair}

To determine whether BMP-7-transduced HGFs suspended in collagen hydrogels induce local skeletal regeneration, surgical osteotomies were prepared in rat femurs and implanted with BMP-7- or lacZ-transduced syngeneic rat dermal fibroblasts (RDFs). In these experiments, $10^{4}$ BMP-7- or $10^{6}$ lacZ-transduced RDFs suspended in a collagen hydrogel and absorbed into gelatin sponges were delivered to the respective sites. lacZ-transduced RDFs failed to form bone bridging the defect $(n=3 / 3$; Fig. 4A,C,E). In contrast, BMP-7transduced RDFs induced cartilage and bone formation within the surgical defect within 6 weeks $(n=3 / 3$; Fig. 4B,D,F). The regenerating bone fused with the existing fragments so that the cut ends are not evident 


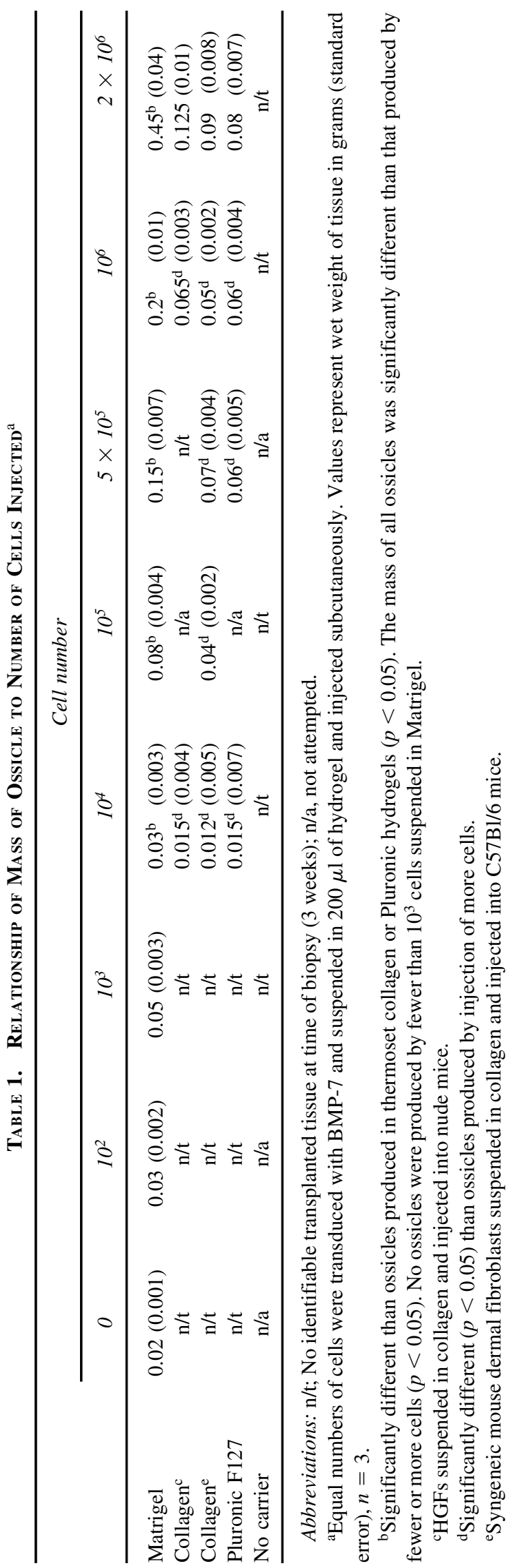




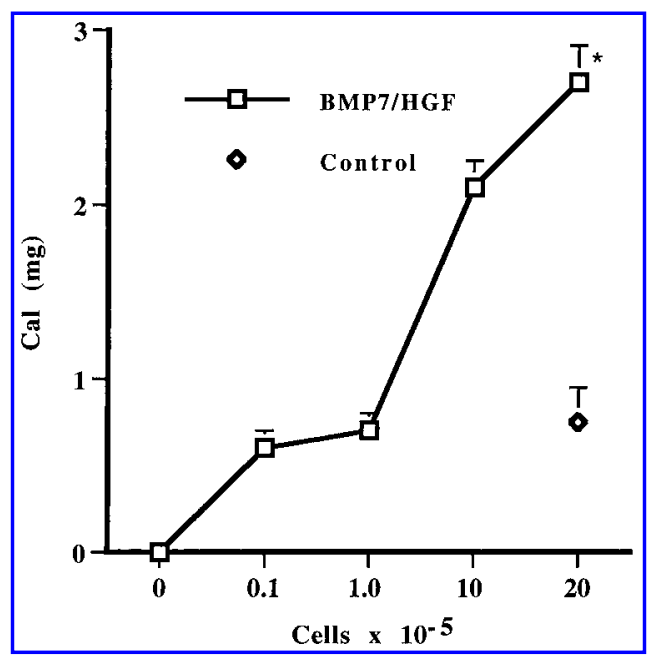

FIG. 2. Calcium content of collagen-suspended,BMP-7-transducedHGF-induced ossicles increases with increasing numbers of implanted cells. No identifiable implanted tissues were recovered from control sites containing fewer than $2 \times 10^{6}$ cells. Calcium content was measured from ashed samples by colorimetric assay according to the manufacturer instructions (Sigma). Data represent means \pm standard deviation $(n=3$, representative of triplicate experiments). Calcium content of samples from $2 \times 10^{6}$ cells is significantly different from control ( ${ }^{*} p<0.01$, Student $t$ test for paired samples).

in the histological specimen (Fig. 4B,D). In the lacZ-transduced RDF control experiments, the defect contained fibrous connective tissue with bone formation limited to remodeling the free ends of the bony fragments.

\section{Osteoblastic transformation of transduced fibroblasts}

Our previous data demonstrated that the BMP-transduced fibroblasts as well as the host fibroblasts contributed to the bony matrix, ${ }^{14}$ suggesting that the BMP-7-transduced fibroblasts converted to osteoblasts in vivo. The ossicles formed by transduced fibroblasts developed through a cartilage intermediate that was usually replaced with bone and marrow by $2-3$ weeks (Fig. 3). To demonstrate that implanted cells directly produce cartilage matrix, HGFs cotransduced with lac $Z$ and BMP-7 were injected subcutaneously and the

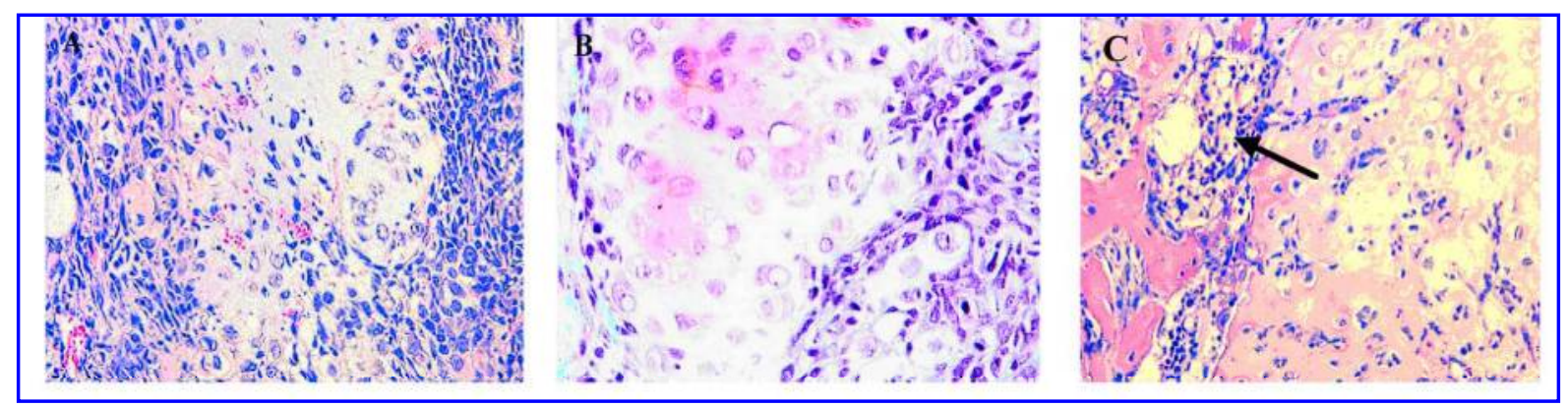

FIG. 3. BMP-7-transduced fibroblasts induce bone via an endochondral-like process in vivo. BMP-7-transduced C57BL/6 dermal fibroblasts $\left(5 \times 10^{5}\right)$ suspended in type I collagen thermoset hydrogel $(2.5 \mathrm{mg} / \mathrm{mL}$, final concentration) were injected subcutaneously into C57BL/6 mice and the resulting tissue was harvested after 3 days (A), 6 days (B), and 14 days (C). (A, C) Stained with H\&E; (B) stained with Safranin O, which stains cartilage proteoglycans red. Developing marrow is evident by 14 days (arrow, C), concomitant with the conversion of the cartilage to a bony matrix. Original magnification, $\times 100$. 


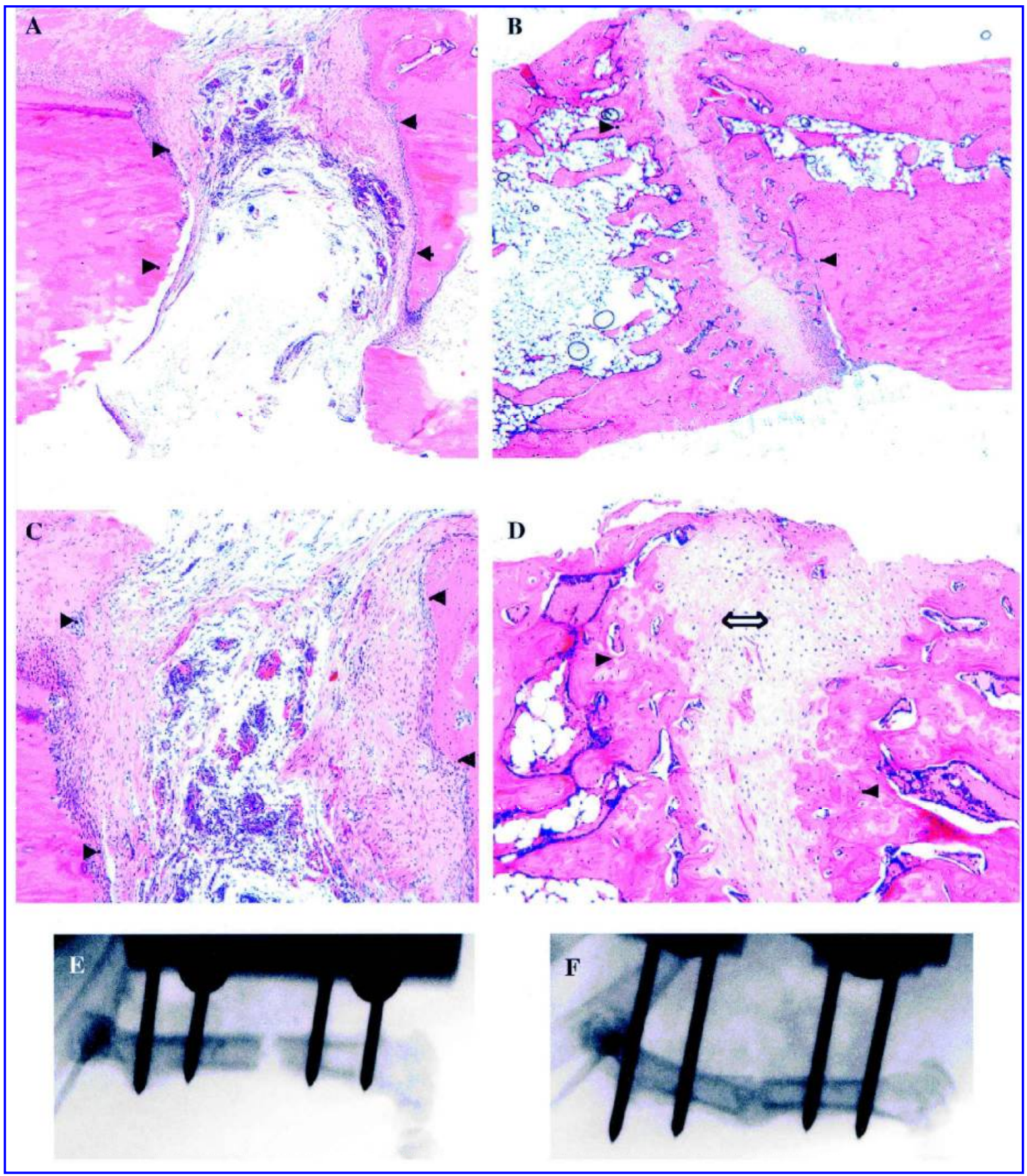

FIG. 4. BMP-7-transduced syngeneic dermal fibroblasts induce skeletal regeneration. Fischer rat dermal fibroblasts were transduced with $\operatorname{lac} Z(\mathbf{A}, \mathbf{B} ; n=3)$ or BMP-7 $(\mathbf{C}, \mathbf{D} ; n=3)$ and suspended at $10^{6}$ or $10^{4}$ cells $/ 30 \mu \mathrm{L}$ of collagen hydrogel, respectively, absorbed to $5 \times 5 \times 5 \mathrm{~mm}$ pieces of gelatin sponge, and implanted into 3-mm surgical osteotomies in Fischer rat femurs for 6 weeks. Histologic analyses revealed only fibrous connective tissue forming in osteotomies implanted with lacZ-transduced RDFs (A, C; arrowheads mark bony margins); whereas bone (arrowheads) and cartilage (double arrow) formed in lesions implanted with BMP-7-transduced RDFs (B, D). Radiographs of lacZ control (E) and BMP-7-transduced $(\mathbf{F})$ treated bones were taken after 6 weeks, just before sacrifice. Original magnification: $(\mathbf{A}, \mathbf{B}) \times 10 ;(\mathbf{C}, \mathbf{D}) \times 50$. 
resultant tissue was analyzed after 7 days. BMP-7- and LacZ-producing cells are directly associated with and surrounded by the developing matrix (Fig. 5A,B) during the cartilagenous phase. These observations reinforce our previously reported data, ${ }^{14}$ that ex vivo BMP-transduced fibroblasts directly contribute to extracellular matrix production. As a definitive test to demonstrate the osteoblastic conversion of fibroblasts by ruling out a contribution by host cells, BMP-7-transduced HGF-collagen suspensions were sealed within diffusion chambers and implanted subcutaneously. A mineralized matrix formed within sealed diffusion chambers containing BMP-7-transduced HGF-collagen composites but not nontransduced fibroblastcollagen composites within 6 weeks $(n=9$, six positive; Fig. 6A-C). Similar tissues were obtained from chambers containing control nontransduced neonatal mouse calvarial-derived periosteal cells (Fig. 6D). The host dermis was in close contact with the diffusion chamber at harvest, with little to no inflammation evident. Extensive histologic analysis of the tissue formed within the chambers (Fig. 5A,C) failed to detect any evidence of host tissue contamination. In striking contrast to results obtained with injection of HGFhydrogel composites (Figs. 2 and 4), no evidence of angiogenesis or marrow formation was evident in the tissues harvested from the diffusion chambers.

\section{DISCUSSION}

Among the pleiotropic effects of BMPs is their remarkable capacity to induce endochondral-like bone formation in nonosteogenic tissues such as gingiva (R.B. Rutherford, unpublished observations), skin, or muscle (reviewed by Kingsley ${ }^{15}$ and $\operatorname{Reddi}^{21,22}$ ). Our data reveal that osteogenesis induced by ex vivo BMPtransduced fibroblasts follows a similar endochondral-like process (Figs. 3-5). The cartilage phase was completely converted to bone even though the rate of conversion appeared to vary with the number of cells implanted and the site of injection (i.e., ectopic vs. orthotopic) (Figs. 3 and 4). However, an organized cartilage-bone interface resembling a growth plate was not detected in any of the several hundred sections examined. These observations reveal that secreted and exogenous recombinant BMP-7 induces bone formation by similar pathways.

One of the major challenges for the tissue engineering of bone is to regulate the amount and form of the new tissue. In our earlier studies, using BMP-transduced fibroblasts and preformed scaffolds, the volume of the ectopic ossicles was largely limited to the volume of the scaffold implanted, and did not vary substantially with the number of attached cells. ${ }^{14}$ Hence the resulting ossicle resembled the size and shape of, and replaced, the implanted cell-laden scaffold. Although robust in producing bone that mimicks a preformed shape, this approach is limited with regard to its ability to deliver osteogenic cells to lesions with complicated anatomy. In the current study, we demonstrate that BMP-7-transduced fibroblasts suspended in thermoset hydrogels from bone in vivo. In this ectopic bone induction assay, the size of the ossicle varied with the number of BMP-7-transduced human cells injected subcutaneously and conformed to the shape

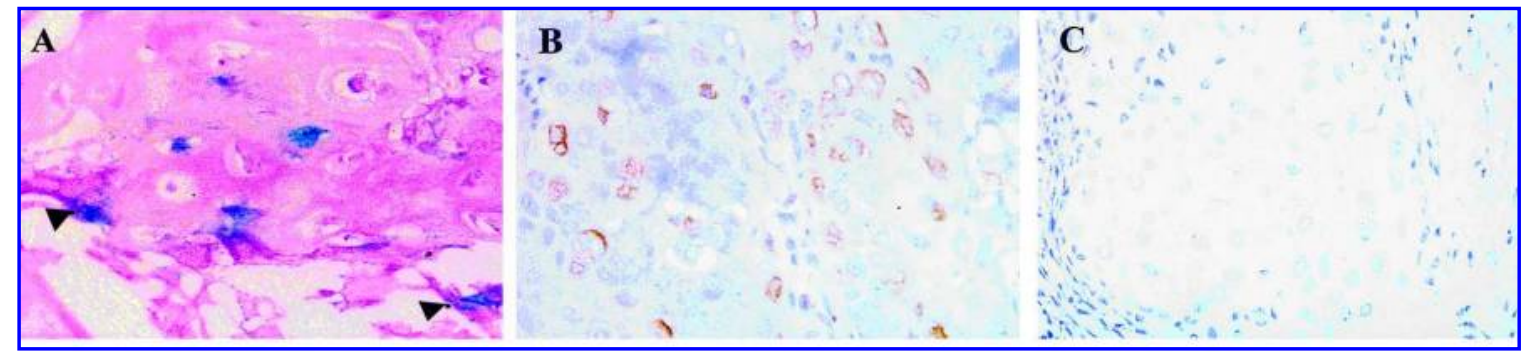

FIG. 5. Ex vivo-transduced fibroblasts are associated with cartilage-like matrix. BMP-7- and lacZ-cotransduced HGFs were suspended in a soluble collagen hydrogel $(2.5 \mathrm{mg} / \mathrm{mL}$, final concentration), injected subcutaneously into NIH III mice, harvested after 7 days, and processed for histochemical and immunocytochemical analyses. (A) LacZ expression by chondroblast-like cells; arrowheads indicate LacZ-producing cells. (B) BMP-7 expression by chondroblast-likecells. (C) Specificity control section [for (B)] developed with anti-BMP-7 antiserum preincubated with BMP-7 ligand. Original magnification: $\times 100$. 

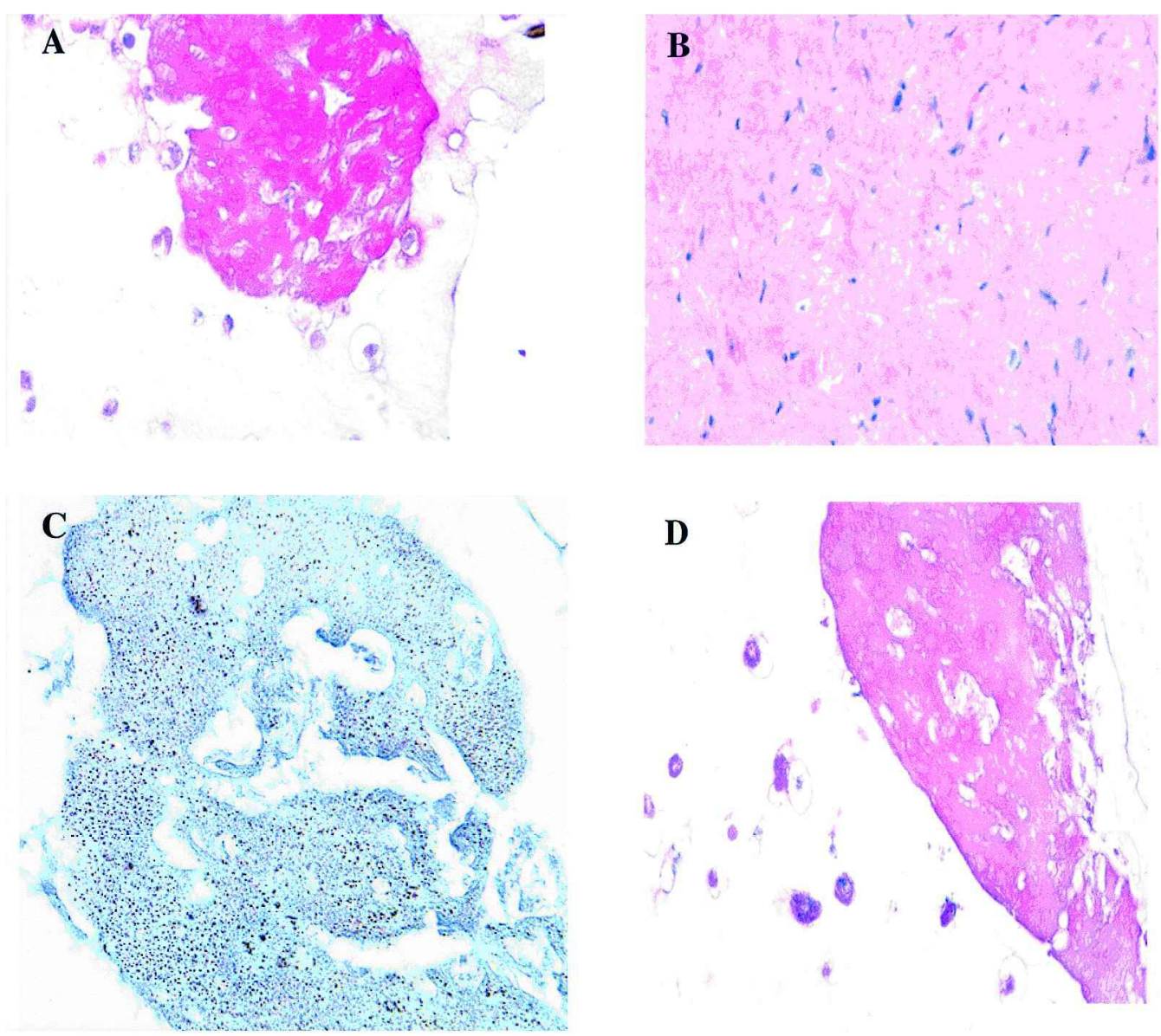

FIG. 6. BMP-7-transduced HGF-collagen gel composites from a cartilage-bone matrix on implantation in immunocompetent mice within diffusion chambers to isolate transduced from host cells. Cells $\left(10^{5}\right)$ in $30 \mu \mathrm{L}$ of collagen solution $(3 \mathrm{mg} / \mathrm{mL})$ were injected into diffusion chambers, gelled, and sealed with nitrocellulose filter paper. The diffusion chambers were implanted subcutaneously in C57BL/6 mice for 6 weeks. Tissues were harvested and processed for histochemical analyses. (A) BMP-7-transduced HGF implant, (B) nontransduced HGF implant, (C) nontransduced neonatal mouse calvarial cell implant, (D) BMP-7-transduced HGF implant stained with von Kossa. Original magnification: $\times 100$.

of the tissue pouch created by injection (data not shown). The size was also dependent on the carrier used but not the immunological competence of the host (Table 1). Hence the type of hydrogel carrier is likely an important factor, because equal numbers of transduced HGFs in equal volumes of hydrogel produce different-sized ossicles depending on the hydrogel used (Table 1). The basis for this differential response has not been studied. The materials tested vary widely in composition and have diverse uses. Matrigel is produced by tumor cells in vitro and contains several extracellular matrix molecules including nonfibrillar type IV collagen and laminin. ${ }^{23}$ The type I collagen contains several cell-binding domains whereas Pluronic, a synthetic surfactant, likely does not. Integrin-mediated cell-extracellular collagen interactions are important in osteoblast differentiation. ${ }^{24-26}$ It is clear that, with fewer than 2 million cells, a carrier is necessary for bone formation (Fig. 2 and Table 1). However, when more than 2 million cells suspended in culture medium are injected, small ossicles develop $(n=4 / 4$; data not shown). The biologic basis for this matrix effect is under investigation.

The use of either porous solid scaffolds or hydrogels alone as carriers for cell implants is not readily adaptable to all potential clinical applications. For example, cells suspended in culture medium are readily 
absorbed to solid porous scaffolds. However, the most gentle manipulation may result in loss of cells from the scaffold. Therefore it may be useful to retain cells within porous, presized scaffolds by first resuspending the cells in an appropriate hydrogel for subsequent loading of the scaffold. Such a method could serve to better retain the cells within the scaffold than if the cells are suspended in a nongelling liquid. Therefore, in these studies, we have tested two strategies for implanting genetically modified fibroblasts. Ex vivotransduced cells suspended in the thermoset hydrogel polymers were directly injected in vivo (Fig. 1) or the hydrogel-cell suspensions were absorbed to preformed porous sponges before surgical implantation (Fig. 4). This approach was utilized in the segmental defect assay, as we anticipated that the hydrogel would not gel quickly enough to form a gelled mass within the segmental defect. Bone formed in both cases, suggesting these delivery modes for BMP-transduced fibroblasts could be useful for localized skeletal regeneration. In the latter case sponges were easily manipulated and placed into the femoral segmental defect without losing absorbed cells. This finding suggests that hydrogel-suspended BMP-transduced fibroblasts could be loaded into porous, custom-designed solid scaffolds before implantation into specific bony sites. ${ }^{27}$ It may not be necessary for the transduced fibroblasts to attach directly to the solid scaffold, providing more latitude in the choice of materials for scaffold construction. However, we have tested only a single solid scaffold (gelatin sponge)-hydrogel (collagen) combination to date. Cells suspended in thermoset hydrogels could be useful in spinal fusion, the restoration of digits, or complex craniofacial structures that may pose anatomical and structural limitations on the implantation of preformed materials.

In our current study, substantial skeletal repair was produced by only $10^{4} \mathrm{BMP}-7$-transduced fibroblasts. Femoral defects contained bone and cartilage that filled the gap after 6 weeks (Fig. 4). Given that more cells produced larger ossicles in the ectopic assay (Table 1), it is likely that more cells would similarly produce a more robust orthotopic response. These data reveal the strong potential for bone regeneration with as few as $10^{4}$ ex vivo BMP-7-transduced, readily available autologous fibroblasts. On the basis of our data (Table 1), it is unlikely that fewer cells will be effective in segmental defects. Other limiting factors affecting the amount of orthotopic bone formed by implantation of transduced fibroblasts have not been further studied.

Most current evidence suggests that reparative cells are recruited from the local tissue (reviewed by Prokop ${ }^{17}$ ). It is therefore likely that cells capable of responding to BMP to initiate bone formation are local. Our hypothesis is that BMP-responsive cells may be propagated from skin and gingiva ex vivo and stimulated to form predetermined amounts of bone on implantation in vivo. In support of this hypothesis we have demonstrated that low-passage human fibroblast strains (from different individual donors) express BMP receptor transcripts in vitro. ${ }^{28}$ HGFs transduced with BMP-7 or cotransduced with BMP-7 and lacZ are found within the developing cartilaginous matrix (Fig. 5), suggesting that the ex vivo BMP-7-transduced cells directly produce bone via an endochondral-like developmental process and that BMP-7 secretion plays a role in cartilage development. Furthermore, BMP-7-transduced HGF-collagen composites, gelled in diffusion chambers and implanted in isolation from direct contact with host tissue, also produce a mineralizing bonelike matrix in vivo (Fig. 6). Such diffusion chamber experiments have been used extensively to determine or confirm the osteoblastic nature of cultured cells. ${ }^{29-31}$ Others have demonstrated bone formation by BMP-transduced fibroblasts. ${ }^{32}$ In addition, the BMP secreted by the implanted transduced cells induces adjacent host cells to form bone. ${ }^{14}$ Taken together, our data reveal that autocrine/intracrine as well as the well-established paracrine modes of signaling operate to form bone on implantation of ex vivo BMPtransduced fibroblasts in vivo. These data support the findings of others, who report an autocrine mode of action for $\mathrm{BMP}^{33}$ but do not distinguish between autocrine or intracrine modes.

Our experiments (Figs. 5 and 6) also demonstrate that BMP-7 transduction of fibroblasts alters the phenotype from fibroblastic to osteoblastic. Consistent with our results, fibroblastic cell lines in culture are known to differentiate to osteoblasts after treatment with exogenous recombinant BMP. ${ }^{34}$ Furthermore, bone marrow and other adult mesenchymally derived tissues possess multipotential cells capable of differentiating along several different pathways, resulting in distinct mesenchymal tissues. ${ }^{35-37}$ Moreover, several studies indicate that adult tissues contain cells capable of differentiating into cells typical of mature tissues derived from a different cell lineage, for example, bone marrow (mesoderm) to neurons (ectoderm). ${ }^{38,39} \mathrm{We}$ have no evidence suggesting that the populations of fibroblasts used in these experiments contain stem cells. It is possible that the fibroblastic cells propagated ex vivo and utilized in these experiments are descendents 
of the cells that respond to exogenous BMP signals in vivo. However, the cells responsive to these exogenous BMP signals have not been identified. It is likely that transduction with BMP-7 alters the transcriptional program and hence the functional phenotype of the transduced fibroblasts.

Ex vivo gene therapy for localized skeletal regeneration, in which the induction of bone formation is by donor cells as well as host cells, could represent a distinct clinical advantage over recombinant protein ${ }^{21}$ or in vivo gene therapy. ${ }^{19,40}$ Both of these approaches require the migration of sufficient numbers of responsive host cells into the lesion. This lengthy process may not readily occur in relatively acellular lesions. In addition, the use of cells, such as dermal or gingival fibroblasts, derived from readily accessible, quickly healing tissues, would be associated with less donor-site morbidity than autologous bony transplants or bone marrow-derived cell therapy. However, a variety of clinical conditions requiring bone regeneration or autologous grafting exist. Further experiments are needed to determine the appropriate and most robust mode of therapy for each.

\section{ACKNOWLEDGMENTS}

We thank Tracy Matson, Rochelle Taylor, and Pat Racenis for excellent technical assistance and Bonnie Knowland and Kathy Sweet for outstanding animal care. We also thank Amy Koh and Laurie McCauley for kindly supplying the mouse osteoblasts. This work was supported by grants from the NIH: DE 12466 (R.B.R.), DE 13386 (R.T.F.), DE 00426 (P.H.K.), AG 00713 (M.M.), and AR 20557 (R.T.).

\section{REFERENCES}

1. Anderson, W.F. Human gene therapy. Nature 392, 25, 1998.

2. Gage, F.H. Cell therapy. Nature 392, 18, 1998.

3. Gazit, D., Turgeman, G., Kelley, P., Wang, E., Jalenak, M., Zilberman, Y., and Moutsatsos, I. Engineered pluripotent mesenchymal cells integrate and differentiate in regenerating bone: a novel cell-mediated gene therapy. J. Gene Med. 1, 121, 1999.

4. Krebsbach, P.H., Kuznetsov, S.A., Satomura, K., Emmons, R.V., Rowe, D.W., and Robey, P.G. Bone formation in vivo: comparison of osteogenesis by transplanted mouse and human marrow stromal fibroblasts. Transplantation 63, 1059, 1997.

5. Breitbart, A.S., Grande, D.A., Kessler, R., Ryaby, J.T., Fitzsimmons, R.J., and Grant, R.T. Tissue engineered bone repair of calvarial defects using cultured periosteal cells. Plast. Reconstr. Surg. 101, 567, 1998.

6. Riew, K.D., Wright, N.M., Cheng, S., Avioli, L.V., and Lou, J. Induction of bone formation using a recombinant adenoviral vector carrying the human BMP-2 gene in a rabbit spinal fusion model. Calcif. Tissue Int. 63, 357, 1998.

7. Breitbart, A.S., Grande, D.A., Mason, J.M., Barcia, M., James, T., and Grant, R.T. Gene-enhanced tissue engineering: applications for bone healing using cultured periosteal cells transduced retrovirally with the BMP-7 gene. Ann. Plast. Surg. 42, 488, 1999.

8. Lieberman, J.R., Le, L.Q., Wu, L., Finerman, G.A., Berk, A., Witte, O.N., and Stevenson, S. Regional gene therapy with a BMP-2-producing murine stromal cell line induces heterotopic and orthotopic bone formation in rodents. J. Orthop. Res. 16, 330, 1998.

9. Lieberman, J.R., Daluiski, A., Stevenson, S., Wu, L., McAllister, P., Lee, Y.P., Kabo, J.M., Finerman, G.A., Berk, A.J., and Witte, O.N. The effect of regional gene therapy with bone morphogenetic protein-2-producing bonemarrow cells on the repair of segmental femoral defects in rats. J. Bone Joint Surg. Am. 81, 905, 1999.

10. Lou, J., Tu, Y., Ludwig, F.J., Zhang, J., and Manske, P. R. Effect of bone morphogenetic protein-12 gene transfer on mesenchymal progenitor cells. Clin. Orthop. 333, 1999.

11. Lou, J., Xu, F., Merkel, K., and Manske, P. Gene therapy: adenovirus-mediated human bone morphogenetic protein-2 gene transfer induces mesenchymal progenitor cell proliferation and differentiation in vitro and bone formation in vivo. J. Orthop. Res. 17, 43, 1999.

12. Lou, J., Tu, Y., Li, S., and Manske, P.R. Involvement of ERK in BMP-2 induced osteoblastic differentiation of mesenchymal progenitor cell line C3H10T1/2. Biochem. Biophys. Res. Commun. 268, 757, 2000.

13. Oakes, D.A., and Lieberman, J.R. Osteoinductive applications of regional gene therapy: ex vivo gene transfer. Clin. Orthop. 379(Suppl.), S101, 2000.

14. Krebsbach, P.H., Gu, K., Franceschi, R.T., and Rutherford, R.B. Gene therapy directed osteogenesis: BMPtransduced human fibroblasts form bone in vivo. Hum. Gene Ther. 11, 1201, 2000. 
15. Kingsley, D.M. What do BMPs do in mammals? Trends Genet. 10, 16, 1994.

16. Reddi, H. Bone and cartilage development. Curr. Opin. Genet. Dev. 4, 737, 1994.

17. Prokop, D.J. Marrow stromal cells as stem cells for nonhematopoietic tissue. Science 276, 71, 1997.

18. Rutherford, R.B., TrailSmith, M.D., Ryan, M.E., and Charette, M.F. Synergistic effects of dexamethasone on platelet-derived growth factor mitogenesis in vitro. Arch. Oral Biol. 37, 139, 1992.

19. Franceschi, R.T., Wang, D., Krebsbach, P.H., and Rutherford, R.B. Gene therapy for bone formation: in vitro and in vivo osteogenic activity of an adenovirus expressing BMP7. J. Cell. Biochem. 78, 476, 2000.

20. Vacanti, C.A., and Upton, J. Tissue-engineered morphogenesis of cartilage and bone by means of cell transplantation using synthetic biodegradable polymer matrices. Clin. Plast. Surg. 21, 445, 1994.

21. Reddi, A.H. Role of morphogenetic proteins in skeletal tissue engineering and regeneration. Nat. Biotechnol. 16, 247, 1998.

22. Reddi, A.H. Regulation of cartilage and bone differentiation by bone morphogenetic protein. Curr. Opin. Cell Biol. 4, 850, 1992.

23. Kleinman, H.K., McGarvey, M.L., Hassell, J.R., Starr, V.L., Cannon, F.B., Laurie, G.W., and Martin, G.R. Basement membrane complexes with biological activity. Biochemistry 25, 312, 1986.

24. Franceschi, R.T., Iyer, B.S., and Cui, Y. Effects of ascorbic acid on collagen matrix formation and osteoblast differentiation in murine MC3T3-E1 cells. J. Bone Miner. Res. 9, 843, 1994.

25. Xiao, G., Wang, D., Benson, M.D., Karsenty, G., and Franceschi, R.T. Role of the $\alpha_{2}$-integrin in osteoblastspecific gene expression and activation of the osf2 transcription factor. J. Biol. Chem. 273, 32988, 1998.

26. Xiao, G., Jiang, D., Thomas, P., Benson, M.D., Guan, K., Karsenty, G., and Franceschi, R.T. MAPK pathways activate and phosphorylate the osteoblast-specific transcription factor, Cbfa1. J. Biol. Chem. 275, 4453, 2000.

27. Levy, S.J., Chu, T.M.G., Halloran, J.W., Feinberg, S.E., and Hollister, S.J. Computed tomography-generated porous hydroxyapatite orbital floor prosthesis as a prototype bioimplant. Am. J. Neuroradiol. 18, 1522, 1997.

28. Gu, K., Smoke, R.H., and Rutherford, R.B. Expression of genes for bone morphogenetic proteins and receptors in human dental pulp. Arch. Oral Biol. 41, 919, 1996.

29. Friedenstein, A.J., Chailakhyan, R.K., and Gerasimov, Y.V. Bone marrow osteogenic stem cells: in vitro cultivation and transplantation in diffusion chambers. Cell Tissue Kinet. 20, 263, 1987.

30. Thomson, B.M., Bennett, J., Dean, V., Triffitt, J., Meikle, M.C., and Loveridge, N. Preliminary characterization of porcine bone marrow stromal cells: skeletogenic potential, colony-forming activity, and response to dexamethasone, transforming growth factor $\beta$, and basic fibroblast growth factor. J. Bone Miner. Res. 8, 1173, 1993.

31. Gundle, R., Joyner, C.J., and Triffitt, J.T. Human bone tissue formation in diffusion chamber culture in vivo by bone-derived cells and marrow stromal fibroblastic cells. Bone 16, 597, 1995.

32. Musgrave, D.S., Bosch, P., Lee, J.Y., Pelinkovic, D., Ghivizzani, S.C., Whalen, J., Niyibizi, C., and Huard, J. Ex vivo gene therapy to produce bone using different cell types. Clin. Orthop. 378, 290, 2000.

33. Shukunami, C., Akiyama, H., Nakamura, T., and Hiraki, Y. Requirement of autocrine signaling by bone morphogenetic protein-4 for chondrogenic differentiation of ATDC5 cells. FEBS Lett. 469, 83, 2000.

34. Wang, E.A., Israel, D.I., Kelly, S., and Luxenberg, D.P. Bone morphogenetic protein-2 causes commitment and differentiation in C3H10T1/2 and 3T3 cells. Growth Factors 9, 57, 1993.

35. Caplan, A.I. Mesenchymal stem cells. J. Orthop. Res. 9, 641, 1991.

36. Pittenger, M.F., Mackay, A.M., Beck, S.C., Jaiswal, R.K., Douglas, R., Mosca, J.D., Moorman, M.A., Simonetti, D.W., Craig, S., and Marshak, D.R. Multilineage potential of adult human mesenchymal stem cells. Science 284, 143, 1999.

37. Krebsbach, P.H., Kuznetsov, S.A., Bianco, P., and Robey, P.G. Bone marrow stromal cells: characterization and clinical application. Crit. Rev. Oral Biol. Med. 10, 165, 1999.

38. Mezey, E., Chandross, K.J., Harta, G., Maki, R.A., and McKercher, S.R. Turning blood into brain: cells bearing neuronal antigens generated in vivo from bone marrow. Science 290, 1779, 2000.

39. Brazelton, T.R., Rossi, F.M.V., Keshet, G.I., and Blau, H.M. From marrow to brain: expression of neuronal phenotypes in adult mice. Science 290, 1775, 2000.

40. Bonadio, J., Smiley, E., Patil, P., and Goldstein, S. Localized, direct plasmid gene delivery in vivo: prolonged therapy results in reproducible tissue regeneration [see comments]. Nat. Med. 5, 753, 1999.

Address reprint requests to: R. Bruce Rutherford, D.D.S., Ph.D. 1011 N. University Ann Arbor, MI 48109-1078 


\section{This article has been cited by:}

1. Richard T. Kao, Shinya Murakami, O. Ross Beirne. 2009. The use of biologic mediators and tissue engineering in dentistry. Periodontology 2000 50:1, 127-153. [CrossRef]

2. Rongying Wang, Yanqiong Zou, Zhen Yuan, Ying Wang, Yuying Chen, Yuanqing Mao, Zhen-An Zhu, Hui Li, Xueming Tang, Jian Lu, Jing Yi. 2009. Autografts and Xenografts of Skin Fibroblasts Delivering BMP-2 Effectively Promote Orthotopic and Ectopic Osteogenesis. The Anatomical Record: Advances in Integrative Anatomy and Evolutionary Biology 292:6, 777-786. [CrossRef]

3. Youngsuk Yi , Kyoung Baek Choi , Chae-Lyul Lim , Jong-Pil Hyun , Hyeon-Youl Lee , Kun Bok Lee, Lillian Yun, Asli Ayverdi , Sally Hwang, Vivian Yip , Moon Jong Noh , Kwan Hee Lee . Irradiated Human Chondrocytes Expressing Bone Morphogenetic Protein 2 Promote Healing of Osteoporotic Bone Fracture in RatsIrradiated Human Chondrocytes Expressing Bone Morphogenetic Protein 2 Promote Healing of Osteoporotic Bone Fracture in Rats. Tissue Engineering Part $A$, ahead of print. [Abstract] [PDF] [PDF Plus]

4. Christopher K. Hee, Steven B. Nicoll. 2009. Endogenous bone morphogenetic proteins mediate 1 $\alpha, 25$-dihydroxyvitamin D 3 -induced expression of osteoblast differentiation markers in human dermal fibroblasts. Journal of Orthopaedic Research 27:2, 162-168. [CrossRef]

5. Emily J. Arnsdorf, Luis M. Jones, Dennis R. Carter, Christopher R. Jacobs . The Periosteum as a Cellular Source for Functional Tissue EngineeringThe Periosteum as a Cellular Source for Functional Tissue Engineering. Tissue Engineering Part A, ahead of print. [Abstract] [PDF] [PDF Plus]

6. Arvydas Usas, Andrew M. Ho , Gregory M. Cooper , Anne Olshanski , Hairong Peng , Johnny Huard . 2009. Bone Regeneration Mediated by BMP4-Expressing Muscle-Derived Stem Cells Is Affected by Delivery SystemBone Regeneration Mediated by BMP4-Expressing Muscle-Derived Stem Cells Is Affected by Delivery System. Tissue Engineering Part A 15:2, 285-293. [Abstract] [PDF] [PDF Plus]

7. Katrin Lorenz, Marit Sicker, Eva Schmelzer, Thomas Rupf, Juergen Salvetter, Michaela Schulz-Siegmund, Augustinus Bader. 2008. Multilineage differentiation potential of human dermal skin-derived fibroblasts. Experimental Dermatology 17:11, 925-932. [CrossRef]

8. W Lattanzi, C Parrilla, A Fetoni, G Logroscino, G Straface, G Pecorini, E Stigliano, A Tampieri, R Bedini, R Pecci, F Michetti, A Gambotto, P D Robbins, E Pola. 2008. Ex vivo-transduced autologous skin fibroblasts expressing human Lim mineralization protein-3 efficiently form new bone in animal models. Gene Therapy 15:19, 1330-1343. [CrossRef]

9. Ke-Rong Dai, Xiao-Ling Zhang, Qin Shi, Julio C Fernandes. 2008. Gene therapy of arthritis and orthopaedic disorders: current experimental approaches in China and in Canada. Expert Opinion on Biological Therapy 8:9, 1337-1346. [CrossRef]

10. Xinping Zhang, Hani A. Awad, Regis J. O’Keefe, Robert E. Guldberg, Edward M. Schwarz. 2008. A Perspective: Engineering Periosteum for Structural Bone Graft Healing. Clinical Orthopaedics and Related Research 466:8, 1777-1787. [CrossRef]

11. Philippe A. Lysy, Françoise Smets, Catherine Sibille, Mustapha Najimi, Etienne M. Sokal. 2007. Human skin fibroblasts: From mesodermal to hepatocyte-like differentiation. Hepatology 46:5, 1574-1585. [CrossRef]

12. Yusuke Hashimoto, Gen Yoshida, Hiromitsu Toyoda, Kunio Takaoka. 2007. Generation of tendon-to-bone interface "enthesis" with use of recombinant BMP-2 in a rabbit model. Journal of Orthopaedic Research 25:11, 1415-1424. [CrossRef] 
13. Keiji Itaka, Shinsuke Ohba, Kanjiro Miyata, Hiroshi Kawaguchi, Kozo Nakamura, Tsuyoshi Takato, Ung-Il Chung, Kazunori Kataoka. 2007. Bone Regeneration by Regulated In Vivo Gene Transfer Using Biocompatible Polyplex Nanomicelles. Molecular Therapy 15:9, 1655-1662. [CrossRef]

14. Jennifer E. Phillips , Robert E. Guldberg , Andrés J. García . 2007. Dermal Fibroblasts Genetically Modified to Express Runx2/Cbfa1 as a Mineralizing Cell Source for Bone Tissue EngineeringDermal Fibroblasts Genetically Modified to Express Runx2/Cbfa1 as a Mineralizing Cell Source for Bone Tissue Engineering. Tissue Engineering 13:8, 2029-2040. [Abstract] [PDF] [PDF Plus]

15. Christian Weinand, Rajiv Gupta, Eli Weinberg, Ijad Madisch, Jesse B. Jupiter, Joseph P. Vacanti. 2007. Human Shaped Thumb Bone Tissue Engineered by Hydrogel-??-Tricalciumphosphate/Poly-??-Caprolactone Scaffolds and Magnetically Sorted Stem Cells. Annals of Plastic Surgery 59:1, 46-52. [CrossRef]

16. Kuo-Yuan Huang, Jing-Jou Yan, Chin-Chiang Hsieh, Ming-Shi Chang, Ruey-Mo Lin. 2007. The In Vivo Biological Effects of Intradiscal Recombinant Human Bone Morphogenetic Protein-2 on the Injured Intervertebral Disc. Spine 32:11, 1174-1180. [CrossRef]

17. Christian Weinand, Rajiv Gupta, Albert Y. Huang, Eli Weinberg, Ijad Madisch, Rameez A. Qudsi , Craig M. Neville, Irina Pomerantseva , Joseph P. Vacanti . 2007. Comparison of Hydrogels in the In Vivo Formation of Tissue-Engineered Bone Using Mesenchymal Stem Cells and Beta-Tricalcium PhosphateComparison of Hydrogels in the In Vivo Formation of Tissue-Engineered Bone Using Mesenchymal Stem Cells and Beta-Tricalcium Phosphate. Tissue Engineering 13:4, 757-765. [Abstract] [PDF] [PDF Plus]

18. Christian Weinand, Rajiv Gupta, Albert Y. Huang, Eli Weinberg, Ijad Madisch, Rameez A. Qudsi, Craig M. Neville, Irina Pomerantseva, Joseph P. Vacanti. 2007. Comparison of Hydrogels in the In Vivo Formation of Tissue-Engineered Bone Using Mesenchymal Stem Cells and Beta-Tricalcium Phosphate. Tissue Engineering, ahead of print070116044221001. [CrossRef]

19. Min Sun Park, Sang-Soo Kim, Seung-Woo Cho, Cha Yong Choi, Byung-Soo Kim. 2006. Enhancement of the osteogenic efficacy of osteoblast transplantation by the sustained delivery of basic fibroblast growth factor. Journal of Biomedical Materials Research Part B: Applied Biomaterials 79B:2, 353-359. [CrossRef]

20. J Kent Leach. 2006. Multifunctional cell-instructive materials for tissue regeneration. Regenerative Medicine 1:4, 447-455. [CrossRef]

21. Sema S. Hakki, Dian Wang, Renny T. Franceschi, Martha J. Somerman. 2006. Bone Sialoprotein Gene Transfer to Periodontal Ligament Cells May Not Be Sufficient to Promote Mineralization In Vitro or In Vivo. Journal of Periodontology 77:2, 167-173. [CrossRef]

22. Judith Cebra-Thomas, Fraser Tan, Seeta Sistla, Eileen Estes, Gunes Bender, Christine Kim, Paul Riccio, Scott F. Gilbert. 2005. How the turtle forms its shell: a paracrine hypothesis of carapace formation. Journal of Experimental Zoology Part B: Molecular and Developmental Evolution 304B:6, 558-569. [CrossRef]

23. RM Schek, JM Taboas, SJ Hollister, PH Krebsbach. 2005. Tissue engineering osteochondral implants for temporomandibular joint repair. Orthodontics and Craniofacial Research 8:4, 313-319. [CrossRef]

24. Conan S. Young, Harutsugi Abukawa, Rose Asrican , Michael Ravens, Maria J. Troulis , Leonard B. Kaban , Joseph P. Vacanti , Pamela C. Yelick . 2005. Tissue-Engineered Hybrid Tooth and BoneTissue-Engineered Hybrid Tooth and Bone. Tissue Engineering 11:9-10, 1599-1610. [Abstract] [PDF] [PDF Plus] 
25. Chia-Ying Lin, Rachel M. Schek, Amit S. Mistry, Xinfeng Shi , Antonios G. Mikos, Paul H. Krebsbach , Scott J. Hollister . 2005. Functional Bone Engineering Using ex Vivo Gene Therapy and Topology-Optimized, Biodegradable Polymer Composite ScaffoldsFunctional Bone Engineering Using ex Vivo Gene Therapy and Topology-Optimized, Biodegradable Polymer Composite Scaffolds. Tissue Engineering 11:9-10, 1589-1598. [Abstract] [PDF] [PDF Plus]

26. Martin Lind, Cody Bünger. 2005. Orthopaedic applications of gene therapy. International Orthopaedics 29:4, 205-209. [CrossRef]

27. Brian Nussenbaum, R Bruce Rutherford, Paul H. Krebsbach. 2005. Bone Regeneration in Cranial Defects Previously Treated with Radiation. The Laryngoscope 115:7, 1170-1177. [CrossRef]

28. Ming Zhao, Zhuoran Zhao, Jeong-Tae Koh, Taocong Jin, Renny T. Franceschi. 2005. Combinatorial gene therapy for bone regeneration: Cooperative interactions between adenovirus vectors expressing bone morphogenetic proteins 2, 4, and 7. Journal of Cellular Biochemistry 95:1, 1-16. [CrossRef]

29. Brett Peterson, Jeffrey Zhang , Roberto Iglesias, Michael Kabo , Marc Hedrick , Prosper Benhaim, Jay R. Lieberman . 2005. Healing of Critically Sized Femoral Defects, Using Genetically Modified Mesenchymal Stem Cells from Human Adipose TissueHealing of Critically Sized Femoral Defects, Using Genetically Modified Mesenchymal Stem Cells from Human Adipose Tissue. Tissue Engineering 11:1-2, 120-129. [Abstract] [PDF] [PDF Plus]

30. Charles A. Gersbach, Benjamin A. Byers, Grace K. Pavlath, Robert E. Guldberg, Andr】s J. GarcDa. 2004. Runx2/Cbfa1-genetically engineered skeletal myoblasts mineralize collagen scaffolds in vitro. Biotechnology and Bioengineering 88:3, 369-378. [CrossRef]

31. Mathew Varkey, Sebastien A Gittens, Hasan Uludag. 2004. Growth factor delivery for bone tissue repair: an update. Expert Opinion on Drug Delivery 1:1, 19-36. [CrossRef]

32. Rachel M. Schek, Juan M. Taboas, Sharon J. Segvich, Scott J. Hollister, Paul H. Krebsbach . 2004. Engineered Osteochondral Grafts Using Biphasic Composite Solid Free-Form Fabricated ScaffoldsEngineered Osteochondral Grafts Using Biphasic Composite Solid Free-Form Fabricated Scaffolds. Tissue Engineering 10:9-10, 1376-1385. [Abstract] [PDF] [PDF Plus]

33. J Kent Leach, David J Mooney. 2004. Bone engineering by controlled delivery of osteoinductive molecules and cells. Expert Opinion on Biological Therapy 4:7, 1015-1027. [CrossRef]

34. Mia Jullig, Wei V. Zhang, N. Susan Stott. 2004. Gene therapy in orthopaedic surgery: the current status. ANZ Journal of Surgery 74:1-2, 46-54. [CrossRef]

35. Emiko Saito, Akira Saito, Masamitsu Kawanami. 2004. Favorable Healing Following Space Creation in rhBMP-2-Induced Periodontal Regeneration of Horizontal Circumferential Defects in Dogs with Experimental Periodontitis. Journal of Periodontology 74:12, 1808-1815. [CrossRef]

36. Brian Nussenbaum , R. Bruce Rutherford, Theodoros N. Teknos , Kenneth J. Dornfeld, Paul H. Krebsbach . 2003. Ex Vivo Gene Therapy for Skeletal Regeneration in Cranial Defects Compromised by Postoperative RadiotherapyEx Vivo Gene Therapy for Skeletal Regeneration in Cranial Defects Compromised by Postoperative Radiotherapy. Human Gene Therapy 14:11, 1107-1115. [Abstract] [PDF] [PDF Plus]

37. I. Kalajzic, Z. Kalajzic, M.M. Hurley, A.C. Lichtler, David W. Rowe. 2003. Stage specific inhibition of osteoblast lineage differentiation by FGF2 and noggin. Journal of Cellular Biochemistry 88:6, 1168-1176. [CrossRef]

38. Q-M. Jin, O. Anusaksathien, S.A. Webb, R.B. Rutherford, W.V. Giannobile. 2003. Gene Therapy of Bone Morphogenetic Protein for Periodontal Tissue Engineering. Journal of Periodontology 74:2, 202-213. [CrossRef] 